\title{
Universal behavior of quantum impurity scattering in a Tomonaga-Luttinger liquid
}

\author{
Yu-Liang Liu \\ Department of Physics, Hong Kong University of Science and Technology, Clear Water Bay, Kowloon, \\ Hong Kong, People's Republic of China
}

(Received 29 July 1998; revised manuscript received 15 October 1998)

\begin{abstract}
Using bosonization and path-integral methods, we study general low-temperature behavior of nonmagnetic and magnetic impurity scattering in Tomonaga-Luttinger liquid, and calculate the electron Green function for a general backward scattering potential. We demonstrate that the electron density of state near the impurity site is suppressed by backward scattering, but it mainly remains invariant far away from the impurity, and that at zero temperature the electrons are completely reflected on the impurity site; the system breaks into two subsystems, but right- and left-moving electron fields have a twisted boundary condition. We also show that a testing charge can only be partially screened by conduction electrons, and in a strong interaction region the impurity susceptibility has a 1/T-type low-temperature behavior. [S0163-1829(99)11111-1]
\end{abstract}

\section{INTRODUCTION}

Recently, considerable efforts have been directed towards the study of the Fermi-edge singularity, ${ }^{1-7}$ the Kondo effect, ${ }^{8-11}$ and the transport properties of one-dimensional (1D) Tomonaga-Luttinger (TL) liquids. ${ }^{12-20}$ The common property of a magnetic and a nonmagnetic impurity scattering in a TL liquid is that there exists backward scattering of electrons on impurity, which drastically influences the lowenergy behavior of the system. The rigorous treatment of the backward scattering is hard work because in the low-energy limit the backward-scattering potential is renormalized to infinity and an usual perturbation expansion method cannot be directly used. As shown in Ref. 12, for a repulsive interacting electronic system, in the low-energy limit, the conduction electrons are completely reflected on the impurity site due to the backward scattering, the system breaks into two subsystems. Under this consideration, the authors in Refs. 21 and 22 studied the low-energy behavior of a TL liquid with an open boundary condition $\psi_{R \sigma}(x)= \pm \psi_{L \sigma}(-x)$ and obtained some results that are consistent with the renormalization-group calculation. ${ }^{12}$ The backward scattering also drastically changes the Fermi-edge singularity, ${ }^{3-7}$ which contributes a finite quantity to the exponent of the x-ray-absorption line-shape function, although between Refs. 3-6 and Ref. 7 there exists some controversy about the contribution size to this exponent by the backward-scattering term. Although the topics have been extensively studied, there are still some theoretical debates about whether the electron's density of state is enhanced or suppressed near and far away from the impurity site, ${ }^{23}$ whether the system breaks into two subsystems at the impurity site with the boundary condition $\psi_{R \sigma}(x)= \pm \psi_{L \sigma}(-x)$ in the low-energy limit, and so on. Because the backward-scattering term is relevant, we need a method to rigorously treat it. In this paper, using an elementary method that can rigorously treat the backwardscattering term, we try to clarify these debating points.

In Secs. II and III, we consider nonmagnetic and magnetic impurity scattering, respectively. Using a unitary transformation, we can eliminate the backward-scattering term (for magnetic impurity, the $J_{2 k_{F}}^{z}$ term), and incorporate its influ- ence on the system into electron interaction terms. In Sec. IV, combining bosonization and path-integral methods, we exactly calculate the Green functions of electrons $\bar{\psi}_{R(L) \sigma}(x)$ and fermions $\bar{\psi}_{1(2) \sigma}(x)$ for generally backward scattering and show that the density of state of electrons near the impurity is suppressed by the backward scattering, while the electron density of state far away from the impurity remains intact. In Sec. V, we show that at zero temperature electrons are completely reflected on the impurity, the system breaks into two subsystems, but the electron fields have a twisted boundary condition. We calculate the exponent of the Fermiedge singularity function of x-ray absorption in Sec. VI. In Secs. VII and VIII, we show that a testing charge is only partially screened by the conduction electrons and study the low-temperature behavior of impurity susceptibility, respectively. We give our conclusion and some discussion in Sec. IX.

\section{NONMAGNETIC IMPURITY SCATTERING}

We consider the following impurity scattering in a general one-dimensional interacting electron system:

$$
\begin{gathered}
H_{T}=H+H_{i m}, \\
H=-i \hbar v_{F} \sum_{\sigma} \int d x\left[\psi_{R \sigma}^{\dagger}(x) \partial_{x} \psi_{R \sigma}(x)-\psi_{L \sigma}^{\dagger}(x) \partial_{x} \psi_{L \sigma}(x)\right] \\
+V_{1} \sum_{\sigma} \int d x \rho_{R \sigma}(x) \rho_{L \sigma}(x) \\
+V_{2} \sum_{\sigma} \int d x \rho_{R \sigma}(x) \rho_{L-\sigma}(x), \\
H_{i m}=\sum_{\sigma} V_{2 k_{F}}\left[\psi_{R \sigma}^{\dagger}(0) \psi_{L \sigma}(0)+\psi_{L \sigma}^{\dagger}(0) \psi_{R \sigma}(0)\right],
\end{gathered}
$$

where $\psi_{R \sigma}(x)$ and $\psi_{R \sigma}^{\dagger}(x)$ are the annihilation and creation field operators of the electrons with spin $\sigma$ that propagate to 
the right with wave vectors approximately $+k_{F} ; \psi_{L \sigma}(x)$ and $\psi_{L \sigma}^{\dagger}(x)$ are the annihilation and creation field operators of left propagating electrons with spin $\sigma$ and wave vectors approximately $-k_{F} ; \rho_{R(L) \sigma}(x)=\psi_{R(L) \sigma}^{\dagger}(x) \psi_{R(L) \sigma}(x)$ are the electron-density operators; the spectrum of the electrons is linearized near the Fermi points and $v_{F}$ is the Fermi velocity. $V_{2 k_{F}}=V\left(k=2 k_{F}\right)$ is the backward-scattering potential of an impurity at $x=0$ on the conduction electrons. For simplicity we have omitted the forward-scattering potential because it is trivial in our transformations. In the bosonic representation of the electron fields ${ }^{24-26} \quad \psi_{R(L) \sigma}(x)=(D /$ $\left.2 \pi \hbar v_{F}\right)^{1 / 2} \exp \left\{-i \Phi_{R(L) \sigma}(x)\right\}$, where $D$ is the bandwidth of the conduction electrons (for simplicity we have neglected the factors $\exp \left\{ \pm i k_{F} x\right\}$ ), the Hamiltonian $H$ can be written as a diagonal form

$$
\begin{aligned}
H= & \frac{\hbar v_{c}}{4 \pi} \int d x \\
& \times\left\{\frac{1}{g_{c}}\left[\partial_{x} \widetilde{\Phi}_{-c}(x)\right]^{2}+g_{c}\left[\partial_{x} \widetilde{\Phi}_{+c}(x)\right]^{2}\right\} \\
& +\frac{\hbar v_{s}}{4 \pi} \int d x\left\{\frac{1}{g_{s}}\left[\partial_{x} \widetilde{\Phi}_{-s}(x)\right]^{2}+g_{s}\left[\partial_{x} \widetilde{\Phi}_{+s}(x)\right]^{2}\right\},
\end{aligned}
$$

where $\quad v_{c}=v_{F}\left(1-\gamma_{c}^{2}\right)^{1 / 2}, v_{s}=v_{F}\left(1-\gamma_{s}^{2}\right)^{1 / 2}, \quad \gamma_{c}=\left(V_{1}\right.$ $\left.+V_{2}\right) / 2 \pi \hbar v_{F}, \quad \gamma_{s}=\left(V_{1}-V_{2}\right) / 2 \pi \hbar v_{F}, \quad g_{c}=\left[\left(1-\gamma_{c}\right) /(1\right.$ $\left.\left.+\gamma_{c}\right)\right]^{1 / 2}, \quad g_{s}=\left[\left(1-\gamma_{s}\right) /\left(1+\gamma_{s}\right)\right]^{1 / 2}, \quad \widetilde{\Phi}_{ \pm c}(x)=\frac{1}{2}\left[\widetilde{\Phi}_{ \pm \uparrow}(x)\right.$ $\left.+\widetilde{\Phi}_{ \pm \downarrow}(x)\right], \quad \widetilde{\Phi}_{ \pm s}(x)=\frac{1}{2}\left[\widetilde{\Phi}_{ \pm \uparrow}(x)-\widetilde{\Phi}_{ \pm \downarrow}(x)\right]$, and $\widetilde{\Phi}_{ \pm \sigma}(x)$ $=\Phi_{R \sigma}(x) \pm \Phi_{L \sigma}(x)$. The impurity scattering term can be written

$$
H_{i m}=\frac{2 D}{\pi \hbar v_{F}} V_{2 k_{F}} \cos \left[\widetilde{\Phi}_{-s}(0)\right] \cos \left[\widetilde{\Phi}_{-c}(0)\right]
$$

which has a conformal dimension $\left(g_{c}+g_{s}\right) / 2$. For the repulsive electron-electron interactions with $V_{1} \geqslant V_{2}$, the dimensionless coupling strength parameters $g_{c}$ and $g_{s}$ are less than one, the backward-scattering term therefore is relevant in the low-energy limit, $V_{2 k_{F}}$ is renormalized to be infinity, ${ }^{12}$ the usual perturbation expansion of $V_{2 k_{F}}$ is invalid.

In order to effectively study the physical property of the system, we define a set of new fermion field operators to diagonalize the Hamiltonian $H_{i m}$,

$$
\begin{aligned}
& \psi_{1 \sigma}(x)=\frac{1}{\sqrt{2}}\left[\psi_{R \sigma}(x)+\psi_{L \sigma}(-x)\right], \\
& \psi_{2 \sigma}(x)=\frac{1}{\sqrt{2}}\left[\psi_{R \sigma}(x)-\psi_{L \sigma}(-x)\right] .
\end{aligned}
$$

It is easy to check that the operators $\psi_{1(2) \sigma}(x)$ satisfy the standard anticommutation relations. In terms of these new fermion fields $\psi_{1(2) \sigma}(x)$, the Hamiltonian (1) can be written as

$$
\begin{aligned}
& H_{1}=-i \hbar v_{F} \sum_{\sigma} \int d x\left[\psi_{1 \sigma}^{\dagger}(x) \partial_{x} \psi_{1 \sigma}(x)+\psi_{2 \sigma}^{\dagger}(x) \partial_{x} \psi_{2 \sigma}(x)\right] \\
&+ \frac{V_{1}}{4} \sum_{\sigma} \int d x\left[\rho_{1 \sigma}(x)+\rho_{2 \sigma}(x)\right]\left[\rho_{1 \sigma}(-x)+\rho_{2 \sigma}(-x)\right] \\
&+ \frac{V_{2}}{4} \sum_{\sigma} \int d x\left[\rho_{1 \sigma}(x)+\rho_{2 \sigma}(x)\right] \\
& \times\left[\rho_{1-\sigma}(-x)+\rho_{2-\sigma}(-x)\right], \\
& H_{2}=-\frac{V_{1}}{4} \sum_{\sigma} \int d x\left[\psi_{1 \sigma}^{\dagger}(x) \psi_{2 \sigma}(x)+\text { H.c. }\right] \\
& \quad \times\left[\psi_{1 \sigma}^{\dagger}(-x) \psi_{2 \sigma}(-x)+\text { H.c. }\right] \\
& \quad-\frac{V_{2}}{4} \sum_{\sigma} \int d x\left[\psi_{1 \sigma}^{\dagger}(x) \psi_{2 \sigma}(x)+\text { H.c. }\right] \\
& \quad \times\left[\psi_{1-\sigma}^{\dagger}(-x) \psi_{2-\sigma}(-x)+\text { H.c. }\right], \\
& H_{i m}=\sum_{\sigma} V_{2 k_{F}}\left[\rho_{1 \sigma}(0)-\rho_{2 \sigma}(0)\right],
\end{aligned}
$$

where $H=H_{1}+H_{2}$. The backward-scattering term $H_{i m}$ becomes a very simple form. In order to study low-energy behavior of the system where the renormalized backwardscattering potential $V_{2 k_{F}}^{R}$ goes to infinity in a low-energy limit, we rewrite the backward-scattering term as

$$
H_{i m}=\sum_{\sigma} \hbar v_{F} \delta\left[\rho_{1 \sigma}(0)-\rho_{2 \sigma}(0)\right],
$$

where $\delta=\arctan \left[V_{2 k_{F}} /\left(\hbar v_{F}\right)\right]$ is a phase shift induced by the backward-scattering potential $V_{2 k_{F}}$. It is reduced to the $H_{i m}$ in Eq. (5) as $V_{2 k_{F}} \rightarrow 0$. Taking this replacement, we can study the property of the system for any value of $V_{2 k_{F}}$. The Hilbert space of the fields $\psi_{R(L) \sigma}(x)$ is different from that of the fields $\psi_{1(2) \sigma}(x)$ in which the backward-scattering term becomes the usual potential scattering in quantum mechanics. However, the transformation (4) is valid for any $V_{2 k_{F}}$; we can take the phase shift $\delta$ as a renormalized quantity varying from zero to $\pm \pi / 2$. This replacement can be justified by the following facts: (a) It is usually used in treatment of a Kondo problem and is proved to be correct. (b) In the interactionfree case, using the Bethe ansatz it can be shown that the impurity scattering potential dependence of ground-state energy is in the form of phase shift $\delta$. (c) In the phase-shift description, at the strong-coupling critical points $\delta^{c}= \pm \pi / 2$ (corresponding to infinity backward-scattering potential) we can easily calculate the Green function and density of state of electrons and show (see below) that they are completely consistent with previous calculations. ${ }^{12,21}$ The bosonic representation of the fermion fields $\psi_{1(2) \sigma}$ can be written as $\psi_{1(2) \sigma}(x)=\left(D / 2 \pi \hbar v_{F}\right)^{1 / 2} \exp \left\{-i \Phi_{1(2) \sigma}(x)\right\}$, where $\rho_{1(2) \sigma}(x)$ $=\psi_{1(2) \sigma}^{\dagger}(x) \psi_{1(2) \sigma}(x)$ are the density operators and are related to the boson field $\Phi_{1(2) \sigma}(x)$ through $\partial_{x} \Phi_{1(2) \sigma}(x)$ $=2 \pi \rho_{1(2) \sigma}(x)$. 
Performing the unitary transformation

$$
U=\exp \left\{i \sum_{\sigma} \frac{\delta}{2 \pi}\left[\Phi_{1 \sigma}(0)-\Phi_{2 \sigma}(0)\right]\right\},
$$

we can have the relation

$$
U^{\dagger}\left(H_{1}+H_{2}+H_{i m}\right) U=H_{1}+U^{\dagger} H_{2} U,
$$

where the unitary transformation of the Hamiltonian $\mathrm{H}_{2}$ can be written as

$$
\begin{aligned}
\bar{H}_{2}= & U^{\dagger} H_{2} U=-\frac{1}{4} \sum_{\sigma} \int d x\left\{V _ { 1 } \left[e^{-i \delta \operatorname{sgn}(x)} \psi_{1 \sigma}^{\dagger}(x) \psi_{2 \sigma}(x)\right.\right. \\
& + \text { H.c. }]\left[e^{i \delta \operatorname{sgn}(x)} \psi_{1 \sigma}^{\dagger}(-x) \psi_{2 \sigma}(-x)+\text { H.c. }\right] \\
& +V_{2}\left[e^{-i \delta \operatorname{sgn}(x)} \psi_{1 \sigma}^{\dagger}(x) \psi_{2 \sigma}(x)+\text { H.c. }\right] \\
& \left.\times\left[e^{i \delta \operatorname{sgn}(x)} \psi_{1-\sigma}^{\dagger}(-x) \psi_{2-\sigma}(-x)+\text { H.c. }\right]\right\}
\end{aligned}
$$

Taking the gauge transformations

$$
\begin{gathered}
\psi_{1 \sigma}(x)=\bar{\psi}_{1 \sigma}(x) e^{i \theta_{1}}, \quad \psi_{2 \sigma}(x)=\bar{\psi}_{2 \sigma}(x) e^{i \theta_{2}}, \\
\theta_{1}-\theta_{2}= \pm \delta
\end{gathered}
$$

which makes $\bar{H}_{2}$ more compact, and leaves $H_{1}$ intact, the Hamiltonian $\bar{H}_{2}=\widetilde{H}_{2}+\widetilde{H}_{2}^{\prime}$ can be rewritten as

$$
\begin{aligned}
\widetilde{H}_{2}= & -\frac{V_{1} \cos (2 \delta)}{4} \sum_{\sigma} \int d x\left[\bar{\psi}_{1 \sigma}^{\dagger}(x) \bar{\psi}_{2 \sigma}(x)+\text { H.c. }\right] \\
& \times\left[\bar{\psi}_{1 \sigma}^{\dagger}(-x) \bar{\psi}_{2 \sigma}(-x)+\text { H.c. }\right] \\
& -\frac{V_{2} \cos (2 \delta)}{4} \sum_{\sigma} \int d x\left[\bar{\psi}_{1 \sigma}^{\dagger}(x) \bar{\psi}_{2 \sigma}(x)+\text { H.c. }\right] \\
& \times\left[\bar{\psi}_{1-\sigma}^{\dagger}(-x) \bar{\psi}_{2-\sigma}(-x)+\text { H.c. }\right], \\
\widetilde{H}_{2}^{\prime}= & i \frac{V_{1} \sin (2 \delta)}{2} \sum_{\sigma} \int_{0}^{\infty} d x\left[\bar{\psi}_{1 \sigma}^{\dagger}(x) \bar{\psi}_{2 \sigma}(x)-\text { H.c. }\right] \\
& \times\left[\bar{\psi}_{1 \sigma}^{\dagger}(-x) \bar{\psi}_{2 \sigma}(-x)+\text { H.c. }\right] \\
& +i \frac{V_{2} \sin (2 \delta)}{2} \sum_{\sigma} \int_{0}^{\infty} d x\left[\bar{\psi}_{1 \sigma}^{\dagger}(x) \bar{\psi}_{2 \sigma}(x)-\text { H.c. }\right] \\
& \times\left[\bar{\psi}_{1-\sigma}^{\dagger}(-x) \bar{\psi}_{2-\sigma}(-x)+\text { H.c. }\right] .
\end{aligned}
$$

To further simplify the Hamiltonian $\bar{H}_{2}$, we can redefine the left- and right-moving electron fields

$$
\begin{aligned}
& \bar{\psi}_{R \sigma}(x)=\frac{1}{\sqrt{2}}\left[\bar{\psi}_{1 \sigma}(x)+\bar{\psi}_{2 \sigma}(x)\right], \\
& \bar{\psi}_{L \sigma}(-x)=\frac{1}{\sqrt{2}}\left[\bar{\psi}_{1 \sigma}(x)-\bar{\psi}_{2 \sigma}(x)\right] .
\end{aligned}
$$

The Hamiltonian $\widetilde{H}_{2}^{\prime}$ becomes

$$
\begin{aligned}
\widetilde{H}_{2}^{\prime}= & \pm i \frac{V_{1} \sin (2 \delta)}{2} \sum_{\sigma} \int_{0}^{\infty} d x\left[\bar{\psi}_{L \sigma}^{\dagger}(-x) \bar{\psi}_{R \sigma}(x)-\text { H.c. }\right] \\
& \times\left[\bar{\rho}_{R \sigma}(-x)-\bar{\rho}_{L \sigma}(x)\right] \pm i \frac{V_{2} \sin (2 \delta)}{2} \sum_{\sigma} \int_{0}^{\infty} d x \\
& \times\left[\bar{\psi}_{L \sigma}^{\dagger}(-x) \bar{\psi}_{R \sigma}(x)-\text { H.c. }\right]\left[\bar{\rho}_{R-\sigma}(-x)-\bar{\rho}_{L-\sigma}(x)\right],
\end{aligned}
$$

where $\bar{\rho}_{R(L) \sigma}(x)=\bar{\psi}_{R(L) \sigma}^{\dagger}(x) \bar{\psi}_{R(L) \sigma}(x)$ are the density operators. The Hamiltonian $\widetilde{H}_{2}^{\prime}$ has a conformal dimension $\Delta>1$ because the field $\bar{\rho}_{R \sigma}(x)-\bar{\rho}_{L \sigma}(-x)$ has the conformal dimension one and the fields $\bar{\psi}_{L \sigma}^{\dagger}(-x) \bar{\psi}_{R \sigma}(x)$ and $\bar{\psi}_{R \sigma}^{\dagger}(x) \bar{\psi}_{L \sigma}(-x)$ have the conformal dimension $\Delta^{\prime}>1$, which can be seen from Eq. (26), and we can neglect it as a first-order approximation. It is zero at the strong-coupling critical points $\delta^{c}= \pm \pi / 2, \widetilde{H}_{2}^{\prime} \equiv 0$. The total Hamiltonian $\bar{H}$ $=H_{1}+\widetilde{H}_{2}$ can be simplified as

$$
\begin{aligned}
\bar{H}= & -i \hbar v_{F} \sum_{\sigma} \int d x\left[\bar{\psi}_{R \sigma}^{\dagger}(x) \partial_{x} \bar{\psi}_{R \sigma}(x)-\bar{\psi}_{L \sigma}^{\dagger}(x) \partial_{x} \bar{\psi}_{L \sigma}(x)\right] \\
& +\frac{V_{1}}{2} \sum_{\sigma} \int d x\left[\alpha \bar{\rho}_{R \sigma}(x) \bar{\rho}_{R \sigma}(-x)+\alpha \bar{\rho}_{L \sigma}(x) \bar{\rho}_{L \sigma}(-x)\right. \\
& \left.+2 \beta \bar{\rho}_{R \sigma}(x) \bar{\rho}_{L \sigma}(x)\right]+\frac{V_{2}}{2} \sum_{\sigma} \int d x\left[\alpha \bar{\rho}_{R \sigma}(x) \bar{\rho}_{R-\sigma}(-x)\right. \\
& \left.+\alpha \bar{\rho}_{L \sigma}(x) \bar{\rho}_{L-\sigma}(-x)+2 \beta \bar{\rho}_{R \sigma}(x) \bar{\rho}_{L-\sigma}(x)\right]
\end{aligned}
$$

where $\alpha=\frac{1}{2}[1-\cos (2 \delta)]$ and $\beta=\frac{1}{2}[1+\cos (2 \delta)]$. For $\delta=0$, without the impurity scattering, we have $\alpha=0$ and $\beta=1$, the Hamiltonian (13) becomes the original one (1). In terms of these new electron fields $\bar{\psi}_{i R(L) \sigma}(x)$, the interaction Hamiltonian $\bar{H}_{I}^{c}$ (last two terms) becomes a very simple form, and the right- and left-moving electrons are completely separated at the critical points $\delta^{c}= \pm \pi / 2$ induced by the backwardscattering potential. However, for a general phase shift $\delta$, the total Hamiltonian (13) becomes less complex and nonlocal. The nonlocality of the interaction terms is the most prominent character of the backward scattering of the conduction electrons on the impurity, which strongly influences the lowenergy behavior of the system.

\section{MAGNETIC IMPURITY SCATTERING}

Now we consider a magnetic impurity scattering. The Kondo interaction term is

$$
\begin{aligned}
H_{K}= & \sum_{i} J_{0}^{i}\left[s_{R}^{i}(0)+s_{L}^{i}(0)\right] S^{i} \\
& +\sum_{i} J_{2 k_{F}}^{i}\left[s_{R L}^{i}(0)+s_{L R}^{i}(0)\right] S^{i},
\end{aligned}
$$

where $\mathbf{S}$ is the impurity spin operator $(S=1 / 2), s_{R(L)}^{i}(0)$ $=\frac{1}{2} \psi_{R(L) \alpha}^{\dagger}(0) \sigma_{\alpha \beta}^{i} \psi_{R(L) \beta}(0), s_{R L}^{i}(0)=\frac{1}{2} \psi_{R \alpha}^{\dagger}(0) \sigma_{\alpha \beta}^{i} \psi_{L \beta}(0)$, 
and $s_{L R}^{i}(0)=\frac{1}{2} \psi_{L \alpha}^{\dagger}(0) \sigma_{\alpha \beta}^{i} \psi_{R \beta}(0)$. In terms of the fields $\psi_{1(2) \sigma}(x)$, it can be written as

$$
H_{K}=\sum_{i} J_{0}^{i}\left[s_{1}^{i}(0)+s_{2}^{i}(0)\right] S^{i}+\sum_{i} J_{2 k_{F}}^{i}\left[s_{1}^{i}(0)-s_{2}^{i}(0)\right] S^{i},
$$

where $s_{1(2)}^{i}(0)=\frac{1}{2} \psi_{1(2) \alpha}^{\dagger}(0) \sigma_{\alpha \beta}^{i} \psi_{1(2) \beta}(0)$. Just as for the nonmagnetic impurity case, we replace the interaction potentials $J_{0}^{z}$ and $J_{2 k_{F}}^{z}$ by the phase shifts $\bar{\delta}$ and $\delta$, respectively, where $\bar{\delta}=\arctan \left[J_{0}^{z} /\left(4 \hbar v_{s}\right)\right]$ and $\delta=\arctan \left[J_{2 k_{F}}^{z} /\left(4 \hbar v_{F}\right)\right]$. Performing the unitary transformation

$$
U^{\prime}=\exp \left\{i \frac{2 g_{s} \bar{\delta}}{\pi} \Phi_{+s}(0) S^{z}+i \frac{2 \delta}{\pi} \Phi_{-s}(0) S^{z}\right\},
$$

where

$$
\Phi_{ \pm s}(0)=\frac{1}{2}\left\{\left[\Phi_{1 \uparrow}(0)-\Phi_{1 \downarrow}(0)\right] \pm\left[\Phi_{2 \uparrow}(0)-\Phi_{2 \downarrow}(0)\right]\right\},
$$

and taking the gauge transformations

$$
\begin{gathered}
\psi_{1 \sigma}(x)=\bar{\psi}_{1 \sigma}(x) e^{i \sigma \theta_{1}}, \quad \psi_{2 \sigma}(x)=\bar{\psi}_{2 \sigma}(x) e^{i \sigma \theta_{2}}, \\
\theta_{1}-\theta_{2}=2 \delta S^{z}
\end{gathered}
$$

where $\sigma=+1$ for spin up $\uparrow$ and $\sigma=-1$ for spin down $\downarrow$, we have the relations $U^{\prime \dagger}\left(H+H_{K}\right) U^{\prime}=H_{1}+\widetilde{H}_{2}+\widetilde{H}_{2}^{\prime \prime}$ $+\bar{H}_{K}$, where the Hamiltonians $H_{1}$ and $\widetilde{H}_{2}$ are the same as that for the nonmagnetic impurity case (5) and (11), and

$$
\begin{aligned}
\bar{H}_{K}= & \frac{J_{1} D}{2 \pi \hbar v_{F}}\left\{e^{-i\left(1+2 g_{s} \bar{\delta} / \pi\right) \Phi_{+s}(0)} e^{-i(1+2 \delta / \pi) \Phi_{-s}(0)} S^{+}\right. \\
& + \text {H.c. }\}+\frac{J_{2} D}{2 \pi \hbar v_{F}} \\
& \times\left\{e^{-i\left(1+2 g_{s} \bar{\delta} / \pi\right) \Phi_{+s}(0)} e^{i(1-2 \delta / \pi) \Phi_{-s}(0)} S^{+}+\text {H.c. }\right\}, \quad(18) \\
\widetilde{H}_{2}^{\prime \prime}= & \pm i \frac{V_{1} \sin (2 \delta)}{2} S^{z} \sum_{\sigma} \int_{0}^{\infty} d x\left[\bar{\psi}_{L \sigma}^{\dagger}(-x) \bar{\psi}_{R \sigma}(x)-\text { H.c. }\right] \\
& \times\left[\bar{\rho}_{R \sigma}(-x)-\bar{\rho}_{L \sigma}(x)\right] \\
& \pm i \frac{V_{2} \sin (2 \delta)}{2} S^{z} \sum_{\sigma} \int_{0}^{\infty} d x\left[\bar{\psi}_{L \sigma}^{\dagger}(-x) \bar{\psi}_{R \sigma}(x)-\text { H.c. }\right] \\
& \times\left[\bar{\rho}_{R-\sigma}(-x)-\bar{\rho}_{L-\sigma}(x)\right],
\end{aligned}
$$

where $J_{1}+J_{2}=J_{0}^{x}=J_{0}^{y}$ and $J_{1}-J_{2}=J_{2 k_{F}}^{x}=J_{2 k_{F}}^{y}$. The Hamiltonian $\widetilde{H}_{2}^{\prime \prime}$, similar to $\bar{H}_{2}^{\prime}$, has a conformal dimension $\Delta^{\prime}$ $>1$, only contributes higher-order correction in the calculation correlation functions, and can be neglected as a firstorder approximation. In the following discussion about the magnetic impurity scattering, we only consider the regions around the fixed points $\delta=0$ and $\delta^{c}= \pm \pi / 2$. At these fixed points the Hamiltonian $\widetilde{H}_{2}^{\prime \prime}$ is zero, and it is reasonable to neglect the Hamiltonian $\widetilde{H}_{2}^{\prime \prime}$ as a first-order approximation. Therefore, except the Hamiltonian $\bar{H}_{K}$ for magnetic impurity scattering, both for the magnetic and nonmagnetic impurity scattering, there exists the same total bulk Hamiltonian $H_{1}$ $+\widetilde{H}_{2}$. It can easily be understood because for the magnetic impurity scattering the $J_{0}^{z}$ and $J_{2 k_{F}}^{z}$ terms are in fact forwardscattering and backward-scattering potential terms, respectively. The low-energy behavior of the system is completely determined by the total bulk Hamiltonian $H_{1}+\widetilde{H}_{2}$. The effect of the impurity scattering on the electrons is reflected on the change of the interactions among the electrons.

\section{CALCULATION OF ELECTRON GREEN FUNCTION}

We first use the Hamiltonian $\bar{H}$ (13) to calculate the Green function of the electron fields $\bar{\psi}_{i R(L) \sigma}(x, \tau)$. The action of the system can be written as

$$
\begin{aligned}
S= & \sum_{\sigma} \int_{0}^{1 /\left(k_{B} T\right)} d \tau \int d x\left\{\bar{\psi}_{R \sigma}^{\dagger}(x, \tau)\left(\partial_{\tau}-i \partial_{x}\right) \bar{\psi}_{R \sigma}(x, \tau)+\bar{\psi}_{L \sigma}^{\dagger}(x, \tau)\left(\partial_{\tau}+i \partial_{x}\right) \bar{\psi}_{L \sigma}(x, \tau)\right\} \\
& -i \sum_{\sigma} \int_{0}^{1 /\left(k_{B} T\right)} d \tau \int d x\left\{\phi_{R \sigma}(x, \tau)\left[\bar{\rho}_{R \sigma}(x, \tau)-\bar{\psi}_{R \sigma}^{\dagger}(x, \tau) \bar{\psi}_{R \sigma}(x, \tau)\right]+\phi_{L \sigma}(x, \tau)\left[\bar{\rho}_{L \sigma}(x, \tau)-\bar{\psi}_{L \sigma}^{\dagger}(x, \tau) \bar{\psi}_{L \sigma}(x, \tau)\right]\right\} \\
& +\frac{V_{1}}{2} \sum_{\sigma} \int_{0}^{1 /\left(k_{B} T\right)} d \tau \int d x\left\{\alpha \bar{\rho}_{R \sigma}(x, \tau) \bar{\rho}_{R \sigma}(-x, \tau)+\alpha \bar{\rho}_{L \sigma}(x, \tau) \bar{\rho}_{L \sigma}(-x, \tau)+2 \beta \bar{\rho}_{R \sigma}(x, \tau) \bar{\rho}_{L \sigma}(x, \tau)\right\} \\
& +\frac{V_{2}}{2} \sum_{\sigma} \int_{0}^{1 /\left(k_{B} T\right)} d \tau \int d x\left\{\alpha \bar{\rho}_{R \sigma}(x, \tau) \bar{\rho}_{R-\sigma}(-x, \tau)+\alpha \bar{\rho}_{L \sigma}(x, \tau) \bar{\rho}_{L-\sigma}(-x, \tau)+2 \beta \bar{\rho}_{R \sigma}(x, \tau) \bar{\rho}_{L-\sigma}(x, \tau)\right\},
\end{aligned}
$$


where $T$ is temperature and the auxiliary fields $\phi_{R(L) \sigma}(x, \tau)$ are the Lagrangians that introduce the constraint conditions $\bar{\rho}_{R(L) \sigma}(x, \tau)=\bar{\psi}_{R(L) \sigma}^{\dagger}(x, \tau) \bar{\psi}_{R(L) \sigma}(x, \tau)$. We have chosen $\hbar$ $=v_{F}=1$. The Green function of the electrons $\bar{\psi}_{R(L) \sigma}(x, \tau)$ can be written as

$$
\begin{aligned}
& G_{R(L) \sigma}\left(x, \tau ; x^{\prime}, \tau^{\prime}\right) \\
&=\frac{1}{\Omega} \int \prod D \bar{\psi} \prod D \bar{\psi}^{\dagger} \prod D \bar{\rho} \prod D \phi \bar{\psi}_{R(L) \sigma}^{\dagger} \\
& \times\left(x^{\prime}, \tau^{\prime}\right) \bar{\psi}_{R(L) \sigma}(x, \tau) e^{-S} \\
&= \frac{1}{\Omega} \int \prod D \bar{\psi} \prod D \bar{\psi}^{\dagger} \prod D \bar{\rho} \prod D \phi \\
& \times G_{R(L) \sigma}\left(x, \tau ; x^{\prime}, \tau^{\prime},[\phi]\right) e^{-S} \\
&= \frac{1}{\Omega} \int \prod_{D} D \bar{\psi} \prod \bar{\psi}^{\dagger} \prod D \bar{\rho} \prod D \phi \\
& \times G_{R(L) \sigma}\left(x, \tau ; x^{\prime}, \tau^{\prime},[\rho]\right) e^{-S},
\end{aligned}
$$

where $\Omega=\int \Pi D \bar{\psi} \Pi D \bar{\psi}^{\dagger} \Pi D \bar{\rho} \Pi D \phi e^{-S}$ is the partition functional of the system, $\Pi D X=\prod_{\sigma} D X_{R \sigma}(x, \tau) D X_{L \sigma}(x, \tau)$, where $X=\left(\bar{\psi}^{\dagger}, \bar{\psi}, \bar{\rho}, \phi\right)$. The Green function $G_{R(L) \sigma}\left(x, \tau ; x^{\prime}, \tau^{\prime},[\rho]\right)$ can be obtained by using the boson representation of the electron fields $\bar{\psi}_{R(L) \sigma}(x, \tau)$. The Green functions $G_{R(L) \sigma}\left(x, \tau ; x^{\prime}, \tau^{\prime},[\phi]\right)$ satisfy the equation

$$
\begin{aligned}
& {\left[\partial_{\tau} \mp i \partial_{x}+i \phi_{R(L) \sigma}(x, \tau)\right] G_{R(L) \sigma}\left(x, \tau ; x^{\prime}, \tau^{\prime},[\phi]\right)} \\
& \quad=-\delta\left(x-x^{\prime}\right) \delta\left(\tau-\tau^{\prime}\right) .
\end{aligned}
$$

Because this is the first-order linear differential equation, we can take the following factorization ansatz ${ }^{27}$

$$
\begin{aligned}
G_{R(L) \sigma}\left(x, \tau ; x^{\prime}, \tau^{\prime},[\phi]\right)= & G_{R(L) \sigma}^{0}\left(x-x^{\prime}, \tau-\tau^{\prime}\right) \\
& \times \exp \left\{f_{R(L) \sigma}(x, \tau,[\phi])\right. \\
& \left.-f_{R(L) \sigma}\left(x^{\prime}, \tau^{\prime},[\phi]\right)\right\},
\end{aligned}
$$

where $\left[\partial_{\tau} \mp i \partial_{x}\right] G_{R(L) \sigma}^{0}(x, \tau)=-\delta(x) \delta(\tau)$ and the fields $f_{R(L) \sigma}(x, \tau,[\phi])$ satisfy the equation

$$
\left[\partial_{\tau} \mp i \partial_{x}\right] f_{R(L) \sigma}(x, \tau,[\phi])=-i \phi_{R(L) \sigma}(x, \tau),
$$

which can be easily $\operatorname{solved}^{28} f_{R(L) \sigma}(x, \tau,[\phi])$ $=k_{B} T \Sigma_{n} \int(d p / 2 \pi) f_{R(L) \sigma}\left(p, \omega_{n},[\phi]\right) e^{i\left(p x-\omega_{n} \tau\right)}$, where $f_{R(L) \sigma}\left(p, \omega_{n},[\phi]\right)=i \phi_{R(L) \sigma}\left(p, \omega_{n}\right) /\left(i \omega_{n} \mp p\right)$. Therefore, in order to calculate the Green function $G_{R(L) \sigma}\left(x, \tau ; x^{\prime}, \tau^{\prime}\right)$, we need to know the effective action $S_{e f f}[\bar{\rho}, \phi]$. After introducing the auxiliary fields $\phi_{R(L) \sigma}(x, \tau)$, there is only the quadratic form of the electron fields $\bar{\psi}_{R(L) \sigma}(x, \tau)$ in Eq. (19). We can integrate them out and obtain the potential function $W(x, \tau)=\operatorname{Tr} \ln \left(\partial_{\tau}-i \partial_{x}+i \phi_{R \sigma}\right)+\operatorname{Tr} \ln \left(\partial_{\tau}+i \partial_{x}+i \phi_{L \sigma}\right)$, which can be calculated by using the Green functions (22) $\quad W(x, \tau)=(i / 2)\left[\phi_{R \sigma}(x, \tau) G_{R \sigma}\left(x, \tau ; x^{\prime} \rightarrow x, \tau^{\prime} \rightarrow \tau\right)\right.$ $\left.+\phi_{L \sigma}(x, \tau) G_{L \sigma}\left(x, \tau ; x^{\prime} \rightarrow x, \tau^{\prime} \rightarrow \tau\right)\right]$. After integrating out the fields $\psi_{R(L) \sigma}(x, \tau)$, we can obtain the effective action

$$
\begin{aligned}
S_{e f f}[\bar{\rho}, \phi]= & k_{B} T \sum_{\sigma} \sum_{n} \int \frac{d p}{2 \pi}\left[A_{R}\left|\phi_{R \sigma}\left(p, \omega_{n}\right)\right|^{2}+A_{L}\left|\phi_{L \sigma}\left(p, \omega_{n}\right)\right|^{2}\right]-i \sum_{\sigma} \int_{0}^{1 /\left(k_{B} T\right)} d \tau \int d x\left\{\phi_{R \sigma}(x, \tau) \bar{\rho}_{R \sigma}(x, \tau)\right. \\
& \left.+\phi_{L \sigma}(x, \tau) \bar{\rho}_{L \sigma}(x, \tau)\right\}+\frac{V_{1}}{2} \sum_{\sigma} \int_{0}^{1 /\left(k_{B} T\right)} d \tau \int d x\left\{\alpha \bar{\rho}_{R \sigma}(x, \tau) \bar{\rho}_{R \sigma}(-x, \tau)+\alpha \bar{\rho}_{L \sigma}(x, \tau) \bar{\rho}_{L \sigma}(-x, \tau)\right. \\
& \left.+2 \beta \bar{\rho}_{R \sigma}(x, \tau) \bar{\rho}_{L \sigma}(x, \tau)\right\}+\frac{V_{2}}{2} \sum_{\sigma} \int_{0}^{1 /\left(k_{B} T\right)} d \tau \int d x\left\{\alpha \bar{\rho}_{R \sigma}(x, \tau) \bar{\rho}_{R-\sigma}(-x, \tau)+\alpha \bar{\rho}_{L \sigma}(x, \tau) \bar{\rho}_{L-\sigma}(-x, \tau)\right. \\
& \left.+2 \beta \bar{\rho}_{R \sigma}(x, \tau) \bar{\rho}_{L-\sigma}(x, \tau)\right\}
\end{aligned}
$$

where $A_{R(L)}=\mp(1 / 4 \pi)\left[p /\left(i \omega_{n} \mp p\right)\right]$. Integrating out the auxiliary fields $\phi_{R(L) \sigma}(x, \tau)$, we can obtain the spin and charge collective excitation spectrums

$$
\epsilon_{c}=\left[1-(\alpha \pm \beta)^{2} \gamma_{c}^{2}\right]^{1 / 2} p, \quad \epsilon_{s}=\left[1-(\alpha \pm \beta)^{2} \gamma_{s}^{2}\right]^{1 / 2} p
$$

It is worth noting that the charge and spin spectrums are influenced by the impurity scattering, which induces the exponents of the electron Green function and other correlation functions depending on the phase shift $\delta$. After integrating out the fields $\bar{\rho}_{R(L) \sigma}(x, \tau)$ and $\phi_{R(L) \sigma}(x, \tau)$ in Eq. (20) and taking the Wick rotation $\tau \rightarrow i t, \quad \tau^{\prime} \rightarrow i t^{\prime}$, we can obtain the electron Green function expression $(\Delta t>0)$,

$$
G_{R(L) \sigma}\left(x, t ; x^{\prime}, t^{\prime}\right)=e^{ \pm i k_{F} \Delta x-Q_{R(L)}\left(x, t ; x^{\prime}, t^{\prime}\right)},
$$




$$
\begin{aligned}
Q_{R(L)}\left(x, t ; x^{\prime}, t^{\prime}\right)= & \frac{1}{4} \sum_{j} \ln \left[\left(\Delta x \mp \alpha_{j+} \Delta t \pm i \eta\right)\left(\Delta x \mp \alpha_{j-} \Delta t \pm i \eta\right)\right]+\frac{1}{8} \sum_{j}\left(\frac{1}{\alpha_{j+}}-1\right) \ln \left[\left(\Delta x \pm \alpha_{j+} \Delta t \mp i \eta\right)\right. \\
& \left.\times\left(\Delta x \mp \alpha_{j+} \Delta t \pm i \eta\right)\right]+\frac{1}{8} \sum_{j}\left(\frac{1}{\alpha_{j-}}-1\right) \ln \left[\left(\Delta x \pm \alpha_{j-} \Delta t \mp i \eta\right)\left(\Delta x \mp \alpha_{j-} \Delta t \pm i \eta\right)\right] \\
& +\frac{1}{16} \sum_{j} \frac{\gamma_{j}}{\alpha_{j+}} \ln \left[\frac{\left(x+x^{\prime}+\alpha_{j+} \Delta t-i \eta\right)^{2}\left(x+x^{\prime}-\alpha_{j+} \Delta t+i \eta\right)^{2}}{(2 x+i \eta)(2 x-i \eta)\left(2 x^{\prime}+i \eta\right)\left(2 x^{\prime}-i \eta\right)}\right] \\
& +\frac{\alpha-\beta}{16} \sum_{j} \frac{\gamma_{j}}{\alpha_{j-}} \ln \left[\frac{\left(x+x^{\prime}+\alpha_{j-} \Delta t-i \eta\right)^{2}\left(x+x^{\prime}-\alpha_{j-} \Delta t+i \eta\right)^{2}}{(2 x+i \eta)(2 x-i \eta)\left(2 x^{\prime}+i \eta\right)\left(2 x^{\prime}-i \eta\right)}\right]
\end{aligned}
$$

where $\alpha_{j \pm}^{2}=1-(\alpha \pm \beta)^{2} \gamma_{j}^{2}, j=c, s, \Delta x=x-x^{\prime}, \Delta t=t$ $-t^{\prime}$, and $\eta$ is the ultraviolet cutoff factor that is proportional to $D^{-1}$. It is easy to demonstrate that at the strong-coupling critical points $\delta^{c}= \pm \pi / 2, G_{R(L) \sigma}(0, t ; 0,0) \sim t^{-\left(1 / g_{c}+1 / g_{s}\right) / 2}$, and at $\delta=0, G_{R(L) \sigma}(0, t ; 0,0) \sim t^{-\left(g_{c}+1 / g_{c}+g_{s}+1 / g_{s}\right) / 4}$. The usual duality relation of the correlation exponents near the impurity site $x=0$ between the ultraviolet $(\delta=0)$ and the infrared fixed points $\left(\delta^{c}= \pm \pi / 2\right)$ is also valid even including the spin degrees of the electrons. ${ }^{29}$ However, it is more important that the correlation exponents are dependent on the phase shift $\delta$, which is consistent with that both the charge and spin collective excitation spectrums and the interaction among electrons are altered by the impurity scattering. From Eq. (26) we can obtain the density of state of the electrons that depends on the distance away from the impurity site. At the strong-coupling critical points $\delta^{c}= \pm \pi / 2$, we can obtain the relation

$$
D_{R(L)}^{c}(x, \omega) \sim \begin{cases}\omega^{\left(1 / g_{c}+1 / g_{s}\right) / 2-1}, & x \rightarrow 0 \\ \omega^{\left(g_{c}+1 / g_{c}+g_{s}+1 / g_{s}\right) / 4-1}, & x \rightarrow \infty,\end{cases}
$$

which is consistent with previous calculations. ${ }^{12,21}$ The impurity backward scattering suppresses the density of state of electrons near the impurity site $x=0$ but has little influence on the electron density of state far away from the impurity.

We can also use the Hamiltonians $H_{1}(5)$ and $\widetilde{H}_{2}(11)$ to calculate the Green function of the fermion fields $\bar{\psi}_{1(2) \sigma}(x)$ by using the same method as above. The action of the system can be written as

$$
\begin{aligned}
S^{\prime}= & \sum_{\sigma} \int_{0}^{1 /\left(k_{B} T\right)} d \tau \int d x\left\{\bar{\psi}_{1 \sigma}^{\dagger}(x, \tau)\left(\partial_{\tau}-i \partial_{x}\right) \bar{\psi}_{1 \sigma}(x, \tau) s+\bar{\psi}_{2 \sigma}^{\dagger}(x, \tau)\left(\partial_{\tau}-i \partial_{x}\right) \bar{\psi}_{2 \sigma}(x, \tau)-i \phi_{1 \sigma}(x, \tau)\left[\bar{\rho}_{1 \sigma}(x, \tau)\right.\right. \\
& \left.-\bar{\psi}_{1 \sigma}^{\dagger}(x, \tau) \bar{\psi}_{1 \sigma}(x, \tau)\right]-i \phi_{2 \sigma}(x, \tau)\left[\bar{\rho}_{2 \sigma}(x, \tau)-\bar{\psi}_{2 \sigma}^{\dagger}(x, \tau) \bar{\psi}_{2 \sigma}(x, \tau)\right]-i \lambda_{1 \sigma}(x, \tau)\left[\Gamma_{1 \sigma}(x, \tau)\right. \\
& \left.-\bar{\psi}_{1 \sigma}^{\dagger}(x, \tau) \bar{\psi}_{2 \sigma}(x, \tau)\right]-i \lambda_{2 \sigma}(x, \tau)\left[\Gamma_{2 \sigma}(x, \tau)-\bar{\psi}_{2 \sigma}^{\dagger}(x, \tau) \bar{\psi}_{1 \sigma}(x, \tau)\right]+\frac{V_{1}}{4}\left[\bar{\rho}_{1 \sigma}(x, \tau)+\bar{\rho}_{2 \sigma}(x, \tau)\right] \\
& \times\left[\bar{\rho}_{1 \sigma}(-x, \tau)+\bar{\rho}_{2 \sigma}(-x, \tau)\right]+\frac{V_{2}}{4}\left[\bar{\rho}_{1 \sigma}(x, \tau)+\bar{\rho}_{2 \sigma}(x, \tau)\right]\left[\bar{\rho}_{1-\sigma}(-x, \tau)+\bar{\rho}_{2-\sigma}(-x, \tau)\right] \\
& -\frac{V_{1} \cos (2 \delta)}{4}\left[\Gamma_{1 \sigma}(x, \tau)+\Gamma_{2 \sigma}(x, \tau)\right]\left[\Gamma_{1 \sigma}(-x, \tau)+\Gamma_{2 \sigma}(-x, \tau)\right]-\frac{V_{2} \cos (2 \delta)}{4} \\
& \left.\times\left[\Gamma_{1 \sigma}(x, \tau)+\Gamma_{2 \sigma}(x, \tau)\right]\left[\Gamma_{1-\sigma}(-x, \tau)+\Gamma_{2-\sigma}(-x, \tau)\right]\right\}
\end{aligned}
$$


where the auxiliary fields $\lambda_{1(2) \sigma}(x, \tau)$ introduce the constraint conditions $\Gamma_{1(2) \sigma}(x, \tau)=\bar{\psi}_{1(2) \sigma}^{\dagger}(x, \tau) \bar{\psi}_{2(1) \sigma}(x, \tau)$. The Green functions of the fermions $\bar{\psi}_{R(L) \sigma}(x, \tau)$ can be written as

$$
\begin{aligned}
& G_{11(22) \sigma}\left(x, \tau ; x^{\prime}, \tau^{\prime}\right) \\
& =\frac{1}{\Omega} \int \prod D \bar{\psi} \prod D \bar{\psi}^{\dagger} \prod D \bar{\rho} \prod D \phi \\
& \times \bar{\psi}_{1(2) \sigma}^{\dagger}\left(x^{\prime}, \tau^{\prime}\right) \bar{\psi}_{1(2) \sigma}(x, \tau) e^{-S^{\prime}} \\
& =\frac{1}{\Omega} \int \prod D \bar{\psi} \prod D \bar{\psi}^{\dagger} \prod D \bar{\rho} \prod D \phi \\
& \times G_{11(22) \sigma}\left(x, \tau ; x^{\prime}, \tau^{\prime},[\phi, \lambda]\right) e^{-S^{\prime}},
\end{aligned}
$$

where $G_{11(22) \sigma}\left(x, \tau ; x^{\prime}, \tau^{\prime},[\phi, \lambda]\right)$ satisfy the linear differential equation

$$
\begin{aligned}
& \left(\begin{array}{ll}
\mathcal{D}+i \phi_{\sigma}, & i \lambda_{\sigma} \\
i \lambda_{\sigma}, & \mathcal{D}+i \phi_{\sigma}
\end{array}\right)\left(\begin{array}{ll}
G_{11}, & G_{12} \\
G_{21}, & G_{22}
\end{array}\right) \\
& \quad=-\left(\begin{array}{ll}
\delta\left(x-x^{\prime}\right) \delta\left(\tau-\tau^{\prime}\right), & 0 \\
0, & \delta\left(x-x^{\prime}\right) \delta\left(\tau-\tau^{\prime}\right)
\end{array}\right),
\end{aligned}
$$

where $\mathcal{D}=\partial_{\tau}-i \partial_{x}, G_{i j}=G_{i j}\left(x, \tau ; x^{\prime}, \tau^{\prime},[\phi, \lambda]\right), \quad i, j=1,2$, and we have taken $\phi_{1 \sigma}(x, \tau)=\phi_{2 \sigma}(x, \tau)=\phi_{\sigma}(x, \tau)$, and $\lambda_{1 \sigma}(x, \tau)=\lambda_{2 \sigma}(x, \tau)=\lambda_{\sigma}(x, \tau)$, because in the action $S^{\prime}$ [Eq. (28)] only the terms $\bar{\rho}_{1 \sigma}+\bar{\rho}_{2 \sigma}$ and $\Gamma_{1 \sigma}+\Gamma_{2 \sigma}$ appear, and after integrating out the fields $\bar{\rho}_{1(2) \sigma}(x, \tau)$ and $\Gamma_{1(2) \sigma}(x, \tau)$ we have the relations $\phi_{1 \sigma}(x, \tau)=\phi_{2 \sigma}(x, \tau)$ and $\lambda_{1 \sigma}(x, \tau)=\lambda_{2 \sigma}(x, \tau)$. Using the factorization ansatz

$$
\begin{aligned}
G_{11(22)}= & \frac{1}{2} G^{0}\left(x, \tau ; x^{\prime}, \tau^{\prime}\right)\left[e^{\tilde{f}_{\sigma}(x, \tau)-\tilde{f}_{\sigma}\left(x^{\prime}, \tau^{\prime}\right)}\right. \\
& \left.+e^{\tilde{f}_{\sigma}\left(x^{\prime}, \tau^{\prime}\right)-\tilde{f}_{\sigma}(x, \tau)}\right] e^{f_{\sigma}(x, \tau)-f_{\sigma^{\prime}}\left(x^{\prime}, \tau^{\prime}\right)}, \\
G_{12(21)}= & \frac{1}{2} G^{0}\left(x, \tau ; x^{\prime}, \tau^{\prime}\right)\left[e^{\tilde{f}_{\sigma}(x, \tau)-\tilde{f}_{\sigma}\left(x^{\prime}, \tau^{\prime}\right)}\right. \\
& \left.-e^{\tilde{f}_{\sigma^{\prime}}\left(x^{\prime}, \tau^{\prime}\right)-\tilde{f}_{\sigma}(x, \tau)}\right] e^{f_{\sigma}(x, \tau)-f_{\sigma^{\prime}}\left(x^{\prime}, \tau^{\prime}\right)},
\end{aligned}
$$

where $\mathcal{D} G^{0}\left(x, \tau ; x^{\prime}, \tau^{\prime}\right)=-\delta\left(x-x^{\prime}\right) \delta\left(\tau-\tau^{\prime}\right)$. If the fields $f_{\sigma}(x, \tau)$ and $\widetilde{f}_{\sigma}(x, \tau)$ satisfy the equations

$$
\begin{aligned}
& \mathcal{D} f_{\sigma}(x, \tau)=-i \phi_{\sigma}(x, \tau), \\
& \mathcal{D} \tilde{f}_{\sigma}(x, \tau)=-i \lambda_{\sigma}(x, \tau),
\end{aligned}
$$

we can easily prove that the expression (31) is the exact solution of the Eq. (30). After integrating out the fields $\bar{\psi}_{1(2) \sigma}(x, \tau)$, we can obtain the potential function

$$
\begin{aligned}
W^{\prime}(x, \tau)= & \operatorname{Tr} \ln (M)=i\left[\phi_{\sigma}(x, \tau) G_{11 \sigma}\left(x, \tau ; x^{\prime} \rightarrow x, \tau^{\prime} \rightarrow \tau\right)\right. \\
& \left.+\lambda_{\sigma}(x, \tau) G_{12 \sigma}\left(x, \tau ; x^{\prime} \rightarrow x, \tau^{\prime} \rightarrow \tau\right)\right]
\end{aligned}
$$

where $M_{11(22)}=\mathcal{D}+i \phi_{\sigma}$ and $M_{12(21)}=i \lambda_{\sigma}$. Using the Green functions $G_{11 \sigma}$ and $G_{12 \sigma}$, we can calculate the potential function $W^{\prime}(x, \tau)$. Integrating out the fields $\bar{\rho}_{1(2) \sigma}(x, \tau)$ and $\Gamma_{1(2) \sigma}(x, \tau)$, we can obtain the effective action

$$
\begin{aligned}
S_{e f f}[\phi, \lambda]= & k_{B} T \sum_{n} \int \frac{d p}{2 \pi}\left\{A ( p , \omega _ { n } ) \left[\left|\phi_{c}\left(p, \omega_{n}\right)\right|^{2}\right.\right. \\
& \left.+\left|\phi_{s}\left(p, \omega_{n}\right)\right|^{2}\right]+A\left(p, \omega_{n}\right)\left[\left|\lambda_{c}\left(p, \omega_{n}\right)\right|^{2}\right. \\
& \left.+\left|\lambda_{s}\left(p, \omega_{n}\right)\right|^{2}\right]+\frac{1}{V_{1}+V_{2}} \phi_{c}\left(p,-\omega_{n}\right) \phi_{c}\left(p, \omega_{n}\right) \\
& +\frac{1}{V_{1}-V_{2}} \phi_{s}\left(p,-\omega_{n}\right) \phi_{s}\left(p, \omega_{n}\right) \\
& -\frac{1}{\left(V_{1}+V_{2}\right) \cos (2 \delta)} \lambda_{c}\left(p,-\omega_{n}\right) \lambda_{c}\left(p, \omega_{n}\right) \\
& \left.-\frac{1}{\left(V_{1}-V_{2}\right) \cos (2 \delta)} \lambda_{s}\left(p,-\omega_{n}\right) \lambda_{s}\left(p, \omega_{n}\right)\right\}
\end{aligned}
$$

where

$$
\begin{gathered}
A\left(p, \omega_{n}\right)=(1 / 2 \pi)\left[p /\left(p-i \omega_{n}\right)\right], \\
\phi_{c(s)}\left(p, \omega_{n}\right)=(\sqrt{2} / 2)\left[\phi_{\uparrow}\left(p, \omega_{n}\right) \pm \phi_{\downarrow}\left(p, \omega_{n}\right)\right],
\end{gathered}
$$

and

$$
\lambda_{c(s)}\left(p, \omega_{n}\right)=(\sqrt{2} / 2)\left[\lambda_{\uparrow}\left(p, \omega_{n}\right) \pm \lambda_{\downarrow}\left(p, \omega_{n}\right)\right] .
$$

Using the effective action $S_{e f f}[\phi, \lambda][$ Eq. (33)], we can obtain the relations after taking the Wick rotation $(\Delta t>0)$,

$$
\begin{aligned}
\left\langle e^{f_{\sigma}(x, t)-f_{\sigma}\left(x^{\prime}, t^{\prime}\right)}\right\rangle=[ & \left.\frac{(\Delta x-\Delta t+i \eta)^{2}}{\left(\Delta x-\alpha_{c} \Delta t+i \eta\right)\left(\Delta x-\alpha_{s} \Delta t+i \eta\right)}\right]^{1 / 4} \prod_{j}\left[\left(\Delta x+\alpha_{j} \Delta t-i \eta\right)\left(\Delta x-\alpha_{j} \Delta t+i \eta\right)\right]^{-\mu_{j} / 4} \\
& \times \prod_{j}\left[\frac{\left(x+x^{\prime}+\alpha_{j} \Delta t-i \eta\right)^{2}\left(x+x^{\prime}-\alpha_{j} \Delta t+i \eta\right)^{2}}{(2 x+i \eta)(2 x-i \eta)\left(2 x^{\prime}+i \eta\right)\left(2 x^{\prime}-i \eta\right)}\right]^{-\nu_{j} / 8},
\end{aligned}
$$




$$
\begin{aligned}
\left\langle e^{\left.\tilde{f}_{\sigma}(x, t)-\tilde{f}_{\left.\sigma^{(} x^{\prime}, t^{\prime}\right)}\right\rangle=}\right. & {\left[\frac{(\Delta x-\Delta t+i \eta)^{2}}{\left(\Delta x-\bar{\alpha}_{c} \Delta t+i \eta\right)\left(\Delta x-\bar{\alpha}_{s} \Delta t+i \eta\right)}\right]^{1 / 4} \prod_{j}[(\Delta x} \\
& \left.\left.+\bar{\alpha}_{j} \Delta t-i \eta\right)\left(\Delta x-\bar{\alpha}_{j} \Delta t+i \eta\right)\right]^{-\bar{\mu}_{j} / 4}, \\
& \times \prod_{j}\left[\frac{\left(x+x^{\prime}+\bar{\alpha}_{j} \Delta t-i \eta\right)^{2}\left(x+x^{\prime}-\bar{\alpha}_{j} \Delta t+i \eta\right)^{2}}{(2 x+i \eta)(2 x-i \eta)\left(2 x^{\prime}+i \eta\right)\left(2 x^{\prime}-i \eta\right)}\right]^{-\bar{\nu}_{j} / 8},
\end{aligned}
$$

where $\quad \alpha_{j}^{2}=1-\gamma_{j}^{2}, \quad \bar{\alpha}_{j}^{2}=1-\cos ^{2}(2 \delta) \gamma_{j}^{2}, \mu_{j}=\frac{1}{2}\left(1 / \alpha_{j}-1\right)$, $\bar{\mu}_{j}=\frac{1}{2}\left(1 / \bar{\alpha}_{j}-1\right), \quad \nu_{j}=\gamma_{j} / 2 \alpha_{j}, \quad$ and $\quad \bar{\nu}_{j}=-\gamma_{j} \cos (2 \delta) /$ $2 \bar{\alpha}_{j}$, where $j=c, s$. The Green functions of the fermions $\bar{\psi}_{1(2) \sigma}(x, t)$ can be written as

$$
\begin{aligned}
G_{11(22)}\left(x, t ; x^{\prime}, t^{\prime}\right)= & G^{0}\left(x, t ; x^{\prime}, t^{\prime}\right)\left\langle e^{f_{\sigma}(x, t)-f_{\sigma}\left(x^{\prime}, t^{\prime}\right)}\right\rangle \\
& \times\left\langle e^{\tilde{f}_{\sigma}(x, t)-\tilde{f}_{\sigma^{\prime}}\left(x^{\prime}, t^{\prime}\right)}\right\rangle .
\end{aligned}
$$

In the impurity-free case, $\delta=0$, the Green functions $G_{11(22)}(0, t ; 0,0)$ have the asymptotic form in the long time limit

$$
G_{11(22)}(0, t ; 0,0) \sim t^{-\left(g_{c}+1 / g_{c}\right) / 4-\left(g_{s}+1 / g_{s}\right) / 4} .
$$

At the strong-coupling critical points $\delta^{c}= \pm \pi / 2$, they have the form

$$
G_{11(22)}^{c}(0, t ; 0,0) \sim t^{-1 / 2 g_{c}-1 / 2 g_{s}} .
$$

It would be pointed out that at $\delta=0$ and the strongcoupling critical points $\delta^{c}= \pm \pi / 2$, the Green functions $G_{R(L)}(0, t ; 0,0)$ and $G_{11(22)}(0, t ; 0,0)$ both have the same asymptotic form in the long time limit, which is different from previous mean-field-approximation calculations. From Eq. (38), we can obtain the density of state of the fermions $\bar{\psi}_{1(2) \sigma}(x)$ at the strong-coupling critical points $\delta^{c}= \pm \pi / 2$,

$$
D_{1(2)}^{c}(0, \omega) \sim \omega^{\left(1 / g_{c}+1 / g_{s}\right) / 2-1},
$$

which is consistent with Eq. (27) for the $x \rightarrow 0$ case. It is clearly shown in Eqs. (27) and (39) that the density of state of electrons near the impurity is suppressed by the backward scattering, but the density of state of electrons far away from the impurity remains intact.

\section{SCATTERING OF ELECTRONS ON THE IMPURITY SITE $x=0$}

We now study the scattering of electrons on the impurity site $x=0$. There is some controversy on this topic. One usually believes that at zero temperature the electrons are completely reflected on the impurity site, therefore there exist the boundary conditions $\psi_{R \sigma}(x, t)= \pm \psi_{L \sigma}(-x, t)$, because the backward-scattering potential is renormalized to infinity as temperature goes to zero. However, our exact solutions of the Green functions $G_{11}\left(x, t ; x^{\prime}, t^{\prime}\right)$ and $G_{22}\left(x, t ; x^{\prime}, t^{\prime}\right)$ in Eq. (36) both have the same expressions even at the strong- coupling critical points $\delta^{c}= \pm \pi / 2$. Therefore, at zero temperature even though the electrons are completely reflected on the impurity site, it does not mean that the boundary conditions $\psi_{R \sigma}(x, t)= \pm \psi_{L \sigma}(-x, t)$ are correct. In this section, we give a correct boundary condition that heavily depends on the phase shift $\delta$.

The influence of the impurity scattering on the electron fields $\psi_{R(L) \sigma}(x)$ is determined by the unitary transformation $U$, after simple calculations we can obtain the relations

$$
\begin{aligned}
U^{\dagger} \psi_{R \sigma}(x) U= & \frac{1}{2} e^{i \theta_{1}} e^{i(\delta / 2) \operatorname{sgn}(x)}\left\{\left[1+e^{-i \delta[1+\operatorname{sgn}(x)]}\right] \bar{\psi}_{R \sigma}(x)\right. \\
& \left.+\left[1-e^{-i \delta[1+\operatorname{sgn}(x)]}\right] \bar{\psi}_{L \sigma}(-x)\right\} \\
U^{\dagger} \psi_{L \sigma}(x) U= & \frac{1}{2} e^{i \theta_{1}} e^{-i(\delta / 2) \operatorname{sgn}(x)}\left\{\left[1-e^{i \delta[1-\operatorname{sgn}(x)]}\right] \bar{\psi}_{R \sigma}(-x)\right. \\
& \left.+\left[1+e^{i \delta[1-\operatorname{sgn}(x)]}\right] \bar{\psi}_{L \sigma}(x)\right\},
\end{aligned}
$$

where for simplicity we have taken the gauge parameters $\theta_{1(2)}$ satisfying $\theta_{1}-\theta_{2}=\delta$. In order to show more clearly the influence of the impurity scattering on electron fields, we consider the following two cases. For the case of $x>0$, the relations (40) can be rewritten as

$$
\begin{gathered}
U^{\dagger} \psi_{R \sigma}(x) U=\frac{1}{2} e^{i \theta_{1}} e^{i \delta / 2}\left[\left(1+e^{-i 2 \delta}\right) \bar{\psi}_{R \sigma}(x)\right. \\
\left.+\left(1-e^{-i 2 \delta}\right) \bar{\psi}_{L \sigma}(-x)\right], \\
U^{\dagger} \psi_{L \sigma}(x) U=e^{i \theta_{1}} e^{-i \delta / 2} \bar{\psi}_{L \sigma}(x) .
\end{gathered}
$$

For the case of $x<0$, the relations (40) can be rewritten as

$$
\begin{gathered}
U^{\dagger} \psi_{R \sigma}(x) U=e^{i \theta_{1}} e^{-i \delta / 2} \bar{\psi}_{R \sigma}(x), \\
U^{\dagger} \psi_{L \sigma}(x) U=\frac{1}{2} e^{i \theta_{1}} e^{i \delta / 2}\left[\left(1-e^{i 2 \delta}\right) \bar{\psi}_{R \sigma}(-x)\right. \\
\left.+\left(1+e^{i 2 \delta}\right) \bar{\psi}_{L \sigma}(x)\right] .
\end{gathered}
$$

The physical explanation of Eqs. (41) and (42) is that a rightmoving electron from $-\infty$ to $+\infty$ and a left-moving electron from $+\infty$ to $-\infty$ are reflected at the impurity site $x=0$. For a general phase shift $\delta$, the right- and left-moving electrons are only partially reflected on the impurity. However, at the 
strong-coupling critical points $\delta^{c}= \pm \pi / 2$, the right- and leftmoving electrons are completely reflected on the impurity site. This can be easily shown from Eqs. (41) and (42),

$$
\begin{aligned}
\left.U^{\dagger} \psi_{R \sigma}(x) U\right|_{\delta^{c}} & = \begin{cases}e^{i \theta_{1}} e^{i \pi / 4} \bar{\psi}_{L \sigma}(-x), & x>0 \\
e^{i \theta_{1}} e^{-i \pi / 4} \bar{\psi}_{R \sigma}(x), & x<0 .\end{cases} \\
\left.U^{\dagger} \psi_{L \sigma}(x) U\right|_{\delta^{c}} & = \begin{cases}e^{i \theta_{1}} e^{-i \pi / 4} \bar{\psi}_{L \sigma}(x), & x>0 \\
e^{i \theta_{1}} e^{i \pi / 4} \bar{\psi}_{R \sigma}(-x), & x<0 .\end{cases}
\end{aligned}
$$

It can be easily seen that there exists a relative phase shift $\Delta \delta=\pi / 2$ between in and out electron wave functions as the right- and left-moving electrons are completely reflected on the impurity site $x=0$, which is different from the boundary conditions $\psi_{R \sigma}(x, t)= \pm \psi_{L \sigma}(-x, t)$. Therefore, Eq. (43) means that at zero temperature this infinite one-dimensional system breaks into two half-infinity subsystems at the impurity site $x=0$, but the electron fields have a twisted boundary condition. Equation (43) is consistent with the calculation of the Green function of the fields $\bar{\psi}_{1(2) \sigma}(x, t)$ at the strongcoupling critical points $\delta^{c}= \pm \pi / 2$.

\section{FERMI-EDGE SINGULARITY FUNCTION OF X-RAY ABSORPTION}

There is some debate about the Fermi-edge singularity of $\mathrm{x}$-ray absorption because previous perturbation approximation calculations give different singularity exponents. Now we recalculate the exponent of the Fermi-edge singularity function of $\mathrm{x}$-ray absorption, which is determined by the correlation function

$$
\begin{aligned}
I_{\sigma}(t) & =\left\langle e^{i H t} \psi_{1 \sigma}^{\dagger}(0) e^{-i\left(H+H_{i m}\right) t} \psi_{1 \sigma}(0)\right\rangle \\
& =\left\langle P(t) \bar{\psi}_{1 \sigma}^{\dagger}(0, t) U(t) U^{\dagger}(0) \bar{\psi}_{1 \sigma}(0,0)\right\rangle,
\end{aligned}
$$

where

$$
P(t)=e^{i H t} e^{-i\left(H_{1}+\widetilde{H}_{2}\right) t} \sim 1
$$

and

$$
U(t)=e^{i\left(H_{1}+\widetilde{H}_{2}\right) t} U e^{-i\left(H_{1}+\widetilde{H}_{2}\right) t} .
$$

In order to calculate the correlation function (44), we need to know the correlation functions of the boson fields $\Phi_{ \pm s}(x, t)$ and $\Phi_{ \pm c}(x, t)$ near the impurity site $x=0$, where $\Phi_{ \pm c}(x, t)$ $=\frac{1}{2}\left\{\left[\Phi_{1 \uparrow}(x, t)+\Phi_{1 \downarrow}(x, t)\right] \pm\left[\Phi_{2 \uparrow}(x, t)+\Phi_{2 \downarrow}(x, t)\right]\right\}$. According to the Hamiltonians $H_{1}$ and $\widetilde{H}_{2}$, the correlation functions of the boson fields $\Phi_{+c(s)}(x, t)$ are completely determined by the Hamiltonian

$$
H^{\prime}=\frac{\hbar v_{c}}{4 \pi} \int d x\left[\partial_{x} \Phi_{+c}(x)\right]^{2}+\frac{\hbar v_{s}}{4 \pi} \int d x\left[\partial_{x} \Phi_{+s}(x)\right]^{2},
$$

where $\Phi_{+c}(x)=\cosh \left(\chi_{c}\right) \Phi_{+c}(x)-\sinh \left(\chi_{c}\right) \Phi_{+c}(-x) \quad$ and $\Phi_{+s}(x)=\cosh \left(\chi_{s}\right) \Phi_{+s}(x)-\sinh \left(\chi_{s}\right) \Phi_{+s}(-x)$, where the parameters $\chi_{c(s)}$ are defined as $\tanh \left(2 \chi_{c(s)}\right)=\gamma_{c(s)}$. It is worth noting that the Hamiltonian (45) is independent of the impurity scattering. By simple calculation, we can obtain the correlation functions in the long time limit

$$
\begin{aligned}
& \left\langle e^{-i \Phi_{+c}(0, t)} e^{i \Phi_{+c}(0,0)}\right\rangle \sim t^{-1 / g_{c}}, \\
& \left\langle e^{-i \Phi_{+s}(0, t)} e^{i \Phi_{+s}(0,0)}\right\rangle \sim t^{-1 / g_{s}} .
\end{aligned}
$$

Using the Green functions $G_{11(22)}(0, t ; 0,0)$ and Eq. (46), we can determine the correlation functions of the boson fields $\Phi_{-c(s)}(0, t)$, because the fermion fields $\bar{\psi}_{1(2) \sigma}(x)$ can be written as in terms of the boson fields $\Phi_{ \pm c}(x)$ and $\Phi_{ \pm s}(x)$,

$$
\begin{aligned}
\bar{\psi}_{1(2) \sigma}(x) \sim & \exp \left\{i \frac { 1 } { 2 } \left[\Phi_{+c}(x) \pm \Phi_{-c}(x)+\sigma \Phi_{+s}(x)\right.\right. \\
& \left.\left. \pm \sigma \Phi_{-s}(x)\right]\right\} .
\end{aligned}
$$

Comparing the Green functions $G_{11(22)}(0, t ; 0,0)$ [Eq. (36)] with Eq. (46), we can easily obtain the correlation functions in the long time limit

$$
\begin{aligned}
& \left\langle e^{-i \Phi_{-c}(0, t)} e^{i \Phi_{-c}(0,0)}\right\rangle \sim t^{-\bar{g}_{c}}, \\
& \left\langle e^{-i \Phi_{-s}(0, t)} e^{i \Phi_{-s}(0,0)}\right\rangle \sim t^{-\bar{g}_{s}},
\end{aligned}
$$

where $\bar{g}_{c(s)}=\sqrt{1-\cos (2 \delta) \gamma_{c(s)}} / \sqrt{1+\cos (2 \delta) \gamma_{c(s)}}$. Using Eqs. (46) and (48), we can obtain the Fermi-edge singularity function of x-ray absorption

$$
I_{\sigma}(\omega) \sim \omega^{\kappa}, \quad \kappa=-1+\frac{1}{4}\left[\frac{1}{g_{c}}+\frac{1}{g_{s}}+\left(1-\frac{2|\delta|}{\pi}\right)^{2} \bar{g}_{c}+\bar{g}_{s}\right]
$$

It is worth noting that the exponent of the Fermi-edge singularity function depends on the phase shift $\delta$, therefore the impurity scattering influences its low-energy behavior. At the strong-coupling critical points $\delta^{c}= \pm \pi / 2$, the exponent $\kappa$ takes the value $\kappa^{c}=-1+1 / 4 g_{c}+1 / 2 g_{s}$. For the interactionfree case, $g_{c}=g_{s}=1$, it takes the valve $\kappa^{c}=-1 / 4$. For the spinless repulsive interacting fermion system $\left(V_{2}=0, g_{c}\right.$ $=g_{s}=g$, and $\bar{g}_{c}=\bar{g}_{s}$ ), using the same method as above, at the strong-coupling critical points $\delta^{c}= \pm \pi / 2$, we can easily obtain $\kappa^{c}=-\frac{7}{8}+1 / 2 g$. For a free spinless fermion system, $g=1$, the $\kappa^{c}$ is $-3 / 8$. This Fermi-edge singularity should be seen in future experiments. These results at $\delta^{c}= \pm \pi / 2$ are the same as those in Refs. 3-6 and different from that in Ref. 7 for the interaction-free case. In these two cases, for small repulsive interaction of the electrons, i.e., $g \sim 1$ (spinless), or $g_{c} \sim g_{s} \sim 1$, there is the Fermi-edge singularity produced by the backward scattering of the deep core-level hole. However, a stronger repulsive interaction of electrons will sweep off (i.e., $\kappa \geqslant 0$ ) the Fermi-edge singularity induced by the backward scattering of the deep core-level hole.

\section{FRIEDEL OSCILLATION AND CHARGE NEUTRALITY}

This is another important issue of the impurity scattering in a one-dimensional system that shows new character different from that in a higher-dimensional system, where the 
impurity can be a pointlike testing charge. Now we take the following impurity scattering Hamiltonian

$$
\begin{aligned}
H_{i m}^{\prime}= & U(0) \sum_{\sigma}\left[\rho_{R \sigma}(0)+\rho_{L \sigma}(0)\right]+U\left(2 k_{F}\right)\left[\psi_{R \sigma}^{\dagger}(0) \psi_{L \sigma}(0)\right. \\
& \left.+\psi_{L \sigma}^{\dagger}(0) \psi_{R \sigma}(0)\right],
\end{aligned}
$$

where $U(0)=Q_{\text {test }}\left(V_{1}+V_{2}\right)$ is the forward-scattering potential, $U\left(2 k_{F}\right)=Q_{\text {test }} V_{2 k_{F}}$ is the backward-scattering potential, and $Q_{\text {test }}$ is a test charge residing at $x=0$. Now the unitary transformation $U$ [Eq. (7)] is replaced by

$$
\begin{aligned}
\bar{U}= & \exp \left(i \sum _ { \sigma } \left\{\frac{g_{c} \widetilde{\delta}}{2 \pi}\left[\Phi_{1 \sigma}(0)+\Phi_{2 \sigma}(0)\right]\right.\right. \\
& \left.\left.+\frac{\delta}{2 \pi}\left[\Phi_{1 \sigma}(0)-\Phi_{2 \sigma}(0)\right]\right\}\right),
\end{aligned}
$$

where the phase shifts $\widetilde{\delta}$ and $\delta$ are defined as $\widetilde{\delta}$ $=\arctan \left[U(0) /\left(\hbar v_{c}\right)\right]$ and $\delta=\arctan \left[U\left(2 k_{F}\right) /\left(\hbar v_{F}\right)\right]$. It must be reminded that the forward scattering does not alter the interaction among electrons, therefore the Green functions of electrons we obtained remain invariant after including the forward-scattering term.

The total density field of electrons reads

$$
\begin{aligned}
\rho(x)= & \frac{1}{2} \sum_{\sigma}\left[\rho_{R \sigma}(x)+\rho_{L \sigma}(x)\right] \\
& +\frac{1}{2} \sum_{\sigma}\left[e^{-i 2 k_{F} x} \psi_{R \sigma}^{\dagger}(x) \psi_{L \sigma}(x)\right. \\
& \left.+e^{i 2 k_{F} x} \psi_{L \sigma}^{\dagger}(x) \psi_{R \sigma}(x)\right] .
\end{aligned}
$$

In terms of the electron fields $\bar{\psi}_{R(L) \sigma}(x)$, we can easily obtain the relations that describe the influence of the impurity scattering on the electron density field

$$
\begin{aligned}
\rho_{>}(x)= & \bar{U}^{\dagger} \rho(x \geqslant 0) \bar{U} \\
= & -\frac{g_{c} \widetilde{\delta}}{\pi} \delta(x) \\
& +\frac{1}{2} \sum_{\sigma}\left[\beta \bar{\rho}_{R \sigma}(x)+\bar{\rho}_{L \sigma}(x)+\alpha \bar{\rho}_{L \sigma}(-x)\right] \\
& +i \frac{\sin (2 \delta)}{4}\left[\bar{\psi}_{R \sigma}^{\dagger}(x) \bar{\psi}_{L \sigma}(-x)-\bar{\psi}_{L \sigma}^{\dagger}(-x) \bar{\psi}_{R \sigma}(x)\right] \\
& +\frac{1}{2}\left\{\left[\cos (\delta) \bar{\psi}_{R \sigma}^{\dagger}(x) \bar{\psi}_{L \sigma}(x)-i \sin (\delta) \bar{\psi}_{L \sigma}^{\dagger}(-x) \bar{\psi}_{L \sigma}\right.\right. \\
& \left.\times(-x)] e^{-i 2 k_{F} x-i g_{c}} \tilde{\delta}_{+} \text {H.c. }\right\},
\end{aligned}
$$

$$
\begin{aligned}
\rho_{<}(x)= & \bar{U}^{\dagger} \rho(x<0) \bar{U} \\
= & \frac{1}{2} \sum_{\sigma}\left[\beta \bar{\rho}_{L \sigma}(x)+\bar{\rho}_{R \sigma}(x)+\alpha \bar{\rho}_{R \sigma}(-x)\right] \\
& +i \frac{\sin (2 \delta)}{4}\left[\bar{\psi}_{L \sigma}^{\dagger}(x) \bar{\psi}_{R \sigma}(-x)-\bar{\psi}_{R \sigma}^{\dagger}(-x) \bar{\psi}_{L \sigma}(x)\right] \\
& +\frac{1}{2}\left\{\left[\cos (\delta) \bar{\psi}_{R \sigma}^{\dagger}(x) \bar{\psi}_{L \sigma}(x)+i \sin (\delta) \bar{\psi}_{R \sigma}^{\dagger}(x) \bar{\psi}_{R \sigma}\right.\right. \\
& \left.\times(-x)] e^{-i 2 k_{F} x+i g_{c}} \tilde{\delta}+\text { H.c. }\right\} .
\end{aligned}
$$

At the strong-coupling critical points $\delta^{c}= \pm \pi / 2$, using the Green functions of the electron fields $\bar{\psi}_{R(L) \sigma}(x)$, we can easily obtain the electron density

$$
\left\langle\rho_{>,<}^{c}(x)\right\rangle \sim \frac{\cos \left(2 k_{F} x \pm g_{c} \widetilde{\delta}\right)}{x^{\left(g_{c}+g_{s}\right) / 2}},
$$

which shows the unusual Friedel oscillation different from that in a high-dimensional system. The total charge induced by the testing charge $Q_{\text {test }}$ can be obtained,

$$
\begin{aligned}
Q^{c}= & \int d x\left\langle\bar{U}^{\dagger} \rho(x) \bar{U}\right\rangle=-\frac{g_{c} \widetilde{\delta}}{\pi} \\
& -a \int_{0}^{\infty} d x \frac{\sin \left(g_{c} \widetilde{\delta}\right) \sin \left(2 k_{F} x\right)+\cos \left(g_{c} \widetilde{\delta}\right) \cos \left(2 k_{F} x\right)}{x^{\left(g_{c}+g_{s}\right) / 2}},
\end{aligned}
$$

where $a$ is a constant, and we have chosen $\Sigma_{\sigma} \int d x\left[\bar{\rho}_{R \sigma}(x)\right.$ $\left.+\bar{\rho}_{L \sigma}(x)\right]=0$. In the case of $\widetilde{\delta} \sim 0$, i.e., weak forward scattering, $Q^{c}$ can be rewritten as

$$
\begin{aligned}
Q^{c}= & -\left(1-g_{c}^{2}\right) Q_{\text {test }}\left[1+a \pi \int_{0}^{\infty} d x \frac{\sin \left(2 k_{F} x\right)}{x^{\left(g_{c}+g_{s}\right) / 2}}\right] \\
& -a \int_{0}^{\infty} d x \frac{\cos \left(2 k_{F} x\right)}{x^{\left(g_{c}+g_{s}\right) / 2}} .
\end{aligned}
$$

The last term is derived from the backward scattering, and because we have taken the phase shift $\delta^{c}= \pm \pi / 2$ [corresponding to $Q_{\text {test }} U\left(2 k_{F}\right) \rightarrow \pm \infty$ ], it is independent of the testing charge $Q_{\text {test }}(\neq 0)$. The charge neutrality means that the relation is rigorously satisfied, $Q^{c}=Q_{\text {test }}$. However, Eq. (57) shows that $Q^{c}$ depends on some parameters such as the bandwidth and the size of the system. Therefore, the conduction electrons cannot completely screen the test charge. Some boundary charge is needed to retain the charge neutrality of the system. This conclusion is consistent with the previous calculation. ${ }^{30}$

\section{IMPURITY SUSCEPTIBILITY OF MAGNETIC IMPURITY SCATTERING}

The magnetic impurity scattering is described by the Kondo interaction term, which is different from the nonmagnetic impurity scattering. We cannot use the unitary transfor- 
mation $U^{\prime}$ [Eq. (16)] completely to eliminate the Kondo interaction term. The spin-exchange interaction term $\bar{H}_{K}[\mathrm{Eq}$. (18)] determines the low-energy behavior of the magnetic impurity. Here we only consider the low-temperature dependence of the impurity susceptibility, which is completely determined by the two phase shifts $\bar{\delta}$ and $\delta$. For simplicity, we use a spinless fermion to represent the magnetic impurity spin, $S^{-}=f, S^{+}=f^{\dagger}$, and $S^{z}=f^{\dagger} f-1 / 2$, the Hamiltonian $\bar{H}_{K}[$ Eq. (18)] can be written as

$$
\bar{H}_{K}=K_{1}\left[\Psi^{\dagger}(0) f+f^{\dagger} \Psi(0)\right]+K_{2}\left[\bar{\Psi}^{\dagger}(0) f+f^{\dagger} \Psi(0)\right],
$$

where

$$
\begin{gathered}
K_{1(2)}=J_{1(2)}\left(D / 2 \pi \hbar v_{F}\right)^{1 / 2}, \\
\Psi(0)=\left(D / 2 \pi \hbar v_{F}\right)^{1 / 2} e^{-i\left(1+2 g_{s} \bar{\delta} / \pi\right) \Phi_{+s}(0)} \\
\\
\times e^{-i(1+2 \delta / \pi) \Phi_{-s}(0)},
\end{gathered}
$$

and

$$
\bar{\Psi}(0)=\left(D / 2 \pi \hbar v_{F}\right)^{1 / 2} e^{-i\left(1+2 g_{s} \bar{\delta} / \pi\right) \Phi_{+s}(0)} e^{i(1-2 \delta / \pi) \Phi_{-s}(0)}
$$

are anyon fields that anticommutate with the fermion field $f$. Now we consider three regions determined by the phase shifts $\bar{\delta}$ and $\delta$ under the condition $g_{s(c)} \leqslant 1$, i.e., for the repulsive interacting electron system.

\section{A. The case of $\bar{\delta} \sim 0$ and $\delta \sim \pm \pi / 2$}

In these regions, the physical property of the system is completely determined by the Hamiltonian at the critical fixed points $\bar{\delta}=0$ and $\delta^{c}= \pm \pi / 2$. The Hamiltonian $\bar{H}_{K}$ [Eq. (58)] at these critical fixed points can be rewritten as

$$
\bar{H}_{K}=K\left[\chi^{\dagger}(0) f+f^{\dagger} \chi(0)\right],
$$

where $K=K_{1}$ and $\chi(0)=\Psi(0)$ for $\delta=-\pi / 2$, and $K=K_{2}$ and $\chi(0)=\bar{\Psi}(0)$ for $\delta=\pi / 2$. We have neglected the term with high conformal dimension. According to Eqs. (46) and (59), we can obtain the Green function of the impurity fermion $f$,

$$
\left\langle f^{\dagger}(\omega) f(-\omega)\right\rangle \sim \frac{1}{i \omega-\Sigma(\omega)}, \quad \Sigma(\omega) \sim|\omega|^{-1+1 / g_{s}}
$$

If $g_{s}=1$, i.e., the interaction-free electron system, the selfenergy of the impurity fermion $\Sigma(\omega)$ becomes a constant, and the system becomes a one-channel Kondo problem. If $g_{s}<1 / 2$, i.e., the strong interaction system, the exponent of the self-energy is larger than one, $-1+1 / g_{s}>1$, and in the low-energy limit the self-energy only contributes higherorder correction, therefore it can be neglected, and the impurity becomes free. However, $1 / 2 \leqslant g_{s}<1$, the low-energy behavior of the impurity is determined by the self-energy $\Sigma(\omega)$. Based upon the above discussion, we can obtain the low-temperature dependence of the impurity susceptibility

$$
\chi_{i m}(T) \sim \begin{cases}\text { const, } & g_{s}=1 \\ T^{3-2 / g_{s},}, & 1 / 2 \leqslant g_{s}<1 \\ T^{-1}, & g_{s}<1 / 2 .\end{cases}
$$

Only in the interaction range $1 / 2<g_{s}<1$ does the exponent of the impurity susceptibility depend on the interaction among electrons. In the strong interaction limit, the impurity becomes free.

\section{B. The case of $\bar{\delta} \sim-\pi / 2$ and $\delta \sim \pm \pi / 2$}

In these regions, the low-energy physical property of the impurity is determined by the Hamiltonian $\bar{H}_{K}$ at the critical fixed points $\bar{\delta}^{c}=-\pi / 2$ and $\delta^{c}= \pm \pi / 2$,

$$
\bar{H}_{K}=K\left[\chi^{\dagger}(0) f+f^{\dagger} \chi(0)\right],
$$

where $\chi(0)=\left(D / 2 \pi \hbar v_{F}\right)^{1 / 2} \exp \left\{i\left(1-g_{s}\right) \Phi_{+s}(0)\right\}$ has the conformal dimension $\left(1-g_{s}\right)^{2} /\left(2 g_{s}\right)$. For the interactionfree case, $g_{s}=1$, the excitation spectrum of the impurity fermion opens a gap proportional to $K$ due to the interaction term (62). In the case of $g_{s}<1$, we can obtain the self-energy of the impurity fermion at these critical fixed points

$$
\Sigma(\omega) \sim|\omega|^{-1+\left(1-g_{s}\right)^{2} / g_{s}}
$$

and the low-temperature dependence of the impurity susceptibility

$$
\chi_{i m}(T) \sim \begin{cases}T^{3-2\left(1-g_{s}\right)^{2} / g_{s},} & \bar{g} \leqslant g_{s}<1 \\ T^{-1}, & g_{s}<\bar{g},\end{cases}
$$

where the parameter $\bar{g}$ is determined by the equation ( 1 $-\bar{g})^{2}=2 \bar{g}$.

\section{The case of $\bar{\delta} \sim-\pi / 2$ and $\delta \sim 0$}

The low-energy behavior of the system in this region is determined by the Hamiltonian $\bar{H}_{K}$ at the critical fixed points $\bar{\delta}^{c}=-\pi / 2$ and $\delta=0$,

$$
\bar{H}=K_{1}\left[\chi^{\dagger}(0) f+f^{\dagger} \chi(0)\right]+K_{2}\left[\bar{\chi}^{\dagger}(0) f+f^{\dagger} \bar{\chi}(0)\right],
$$

where $\quad \chi(0)=\left(D / 2 \pi \hbar v_{F}\right)^{1 / 2} \exp \left\{i\left(1-g_{s}\right) \Phi_{+s}(0)+i \Phi_{-s}(0)\right\}$ and $\bar{\chi}(0)=\left(D / 2 \pi \hbar v_{F}\right)^{1 / 2} \exp \left\{i\left(1-g_{s}\right) \Phi_{+s}(0)-i \Phi_{-s}(0)\right\}$. For the interaction-free case, $g_{s}=1$, we have the relation $\chi(0)$ $=\bar{\chi}(0)$. The system becomes an asymmetric two-channel Kondo model if $K_{1} \neq K_{2}$. It is worth noting that $\bar{\delta}=-\pi / 2$ and $\delta=0$ are not stable critical fixed points, because for the repulsive interaction system $\left(g_{s(c)}<1\right)$ the backwardscattering $J_{2 k_{F}}^{z}$ term has the conformal dimension $\left(g_{c}\right.$ $\left.+g_{s}\right) / 2<1$, and it is relevant in terminology of the renormalization group. The backward-scattering potential $J_{2 k_{F}}^{z}$ is renormalized to infinity in the low-energy limit, which corresponds to the phase shift $\delta^{c}= \pm \pi / 2$. However, here we artificially assume that the backward-scattering potential is 
very small at some low-energy region so that we can discuss some low-energy behavior of the impurity near the point $\delta$ $=0$.

In the case of $g_{s}=1$, the system becomes a two-channel Kondo problem. If $K_{1}=K_{2}$, the low-temperature dependence of the impurity susceptibility is ${ }^{31} \chi_{i m}(T) \sim \ln (T)$. If $K_{1} \neq K_{2}$, the asymmetry of the two channels destroys the $\ln (T)$ dependence of the impurity susceptibility and makes it show the low-temperature behavior of the one-channel Kondo problem. ${ }^{32}$ In the case of $g_{s}<1$, the self-energy of the impurity fermion consists of two parts: one is contributed directly by the anyon fields $\chi(0)$ and $\bar{\chi}(0)$, and the other one is from their hybridization,

$$
\begin{gathered}
\Sigma_{1}(\omega) \sim\left(K_{1}^{2}+K_{2}^{2}\right)|\omega|^{\left(1-g_{s}\right)^{2} / g_{s}+g_{s}-1}, \\
\Sigma_{2}(\omega) \sim K_{1} K_{2}|\omega|^{\left(1-g_{s}\right)^{2} / g_{s}-g_{s}-1} .
\end{gathered}
$$

It can be seen that in the range $1 / 4<g_{s}<1$, the impurity susceptibility has the power-law temperature dependence in the low-temperature region. However, in the strong interaction region $g_{s}<1 / 4$, the impurity spin becomes free, and the impurity susceptibility shows the low-temperature behavior $1 / T$. It is worth noting that in the three different regions $A, B$, and $C$, they all show that in the strong repulsive interaction region the impurity spin becomes free, which is the most prominent character of magnetic impurity scattering in a one-dimensional electron system. These results are consistent with previous calculations for a magnetic impurity scattering in the Heisenberg chain. ${ }^{33}$ The physical explanation of the low-temperature power-law behavior of the impurity susceptibility is that the impurity scattering suppresses the density of state of electrons near the impurity site for the repulsive interacting electron system, therefore the impurity spin is only partially screened, which is similar to the nonmagnetic impurity scattering case where the testing charge $Q_{\text {test }}$ is partially screened by conduction electrons.

The impurity susceptibilities in Eqs. (61) and (64) are different from that in Ref. 11. The result in Ref. 11 was obtained by taking the way that for $J_{2}=0$ (here we use present labels) and $V_{1}=V_{2}=0$ the system becomes the usual one-channel Kondo problem. It is well-known that it has an infrared Fermi-liquid fixed point, and they assumed that after switching electron interaction, as $\left\{V_{1}, V_{2}\right\} \rightarrow 0$, the system has the same infrared fixed point as the one-channel Kondo problem. This assumption is crucial, and its correctness is unclear. First, for a one-dimensional interacting electron system, the forward-scattering potential is not generally equal to the backward-scattering one because they satisfy different renormalization group equations in the low-energy limit. Second, for an interaction-free electron system, the backward-scattering term induced by the magnetic impurity is marginal, but for a repulsive interaction electron system, the backward-scattering term is relevant, and the interactionfree system has an infrared fixed point different from that of the interaction system. Generally, there does not exist a principle to guarantee that there is a smooth connection between these two infrared fixed points as the interaction potentials $V_{1}$ and $V_{2}$ go to zero.

\section{CONCLUSION AND DISCUSSION}

Combining the basic path integral and bosonization methods, we have studied the low-energy behavior of the magnetic and nonmagnetic impurity scattering in a onedimensional repulsive interacting electron system (Tomonaga-Luttinger liquid) and discussed some basic and controversial issues of these topics. Due to the linearization of the excitation spectrum of electrons near their two Fermi levels, using the factorization ansatz we have exactly calculated the Green functions of electrons $\bar{\psi}_{R(L) \sigma}(x)$ and fermions $\bar{\psi}_{1(2) \sigma}(x)$ for a general phase shift $\delta$ induced by the backward-scattering potential of the impurity. The influence of the backward scattering on the system is great, because the backward scattering alters the interaction among electrons and makes the exponents of all but density-density correlation functions depend on the phase shift $\delta$. This the most prominent character of the backward scattering in Tomonaga-Luttinger liquid.

Because the backward-scattering term is relevant, any perturbation expansion of this term is hard to give a rigorous description for the system from weak to strong backward scattering; therefore it is not surprising that there are some controversial issues of these topics, such as the density of state of electrons near and far away from the impurity site, the exponent of the Fermi-edge singularity function of x-ray absorption, the low-temperature behavior of the impurity susceptibility, the boundary conditions of electron fields at zero temperature on impurity site, and so on. However, by using the simple unitary and global gauge transformations, the backward-scattering term can be rigorously treated, and its influence on the system is incorporated in the interaction terms among electrons, then using the path-integral method all correlation functions we need can be exactly calculated. Therefore, we believe that our results are correct, and can be used to justify previous results obtained by other methods.

The most important properties of the impurity scattering in Tomonaga-Luttinger liquid are as follows: (a) At zero temperature the electrons are completely reflected on the impurity site $x=0$, the system breaks into two subsystems at $x=0$, but the right- and left-moving electron fields have the twisted boundary condition. (b) The density of state of electrons is suppressed near the impurity site, but it mainly remains invariant far away from the impurity. (c) The exponents of correlation functions, such as the Green functions of electrons $\bar{\psi}_{R(L) \sigma}(x)$ and fermions $\bar{\psi}_{1(2) \sigma}(x)$, depend upon the phase shift $\delta$ induced by backward scattering. (d) In the low-energy limit, the testing charge is only partially screened by the conduction electrons. (e) In the weak repulsive interaction region, the impurity susceptibility has the power-law low-temperature dependence. In the strong repulsive interaction region, the impurity spin becomes free, and the impurity susceptibility has the 1/T-type low-temperature behavior.

\section{ACKNOWLEDGMENTS}

The author would like to thank Dr. T. K. Ng for helpful discussions and acknowledge the support of HKRGC through Grant No. UST6143/97P. 
${ }^{1}$ T. Ogawa, A. Furusaki, and N. Nagaosa, Phys. Rev. Lett. 68, 3638 (1992); A. Furusaki and N. Nagaosa, Phys. Rev. B 47, 3827 (1993).

${ }^{2}$ D. K. K. Lee and Y. Chen, Phys. Rev. Lett. 69, 1399 (1992).

${ }^{3}$ A. O. Gogolin, Phys. Rev. Lett. 71, 2995 (1993).

${ }^{4}$ N. V. Prokof'ev, Phys. Rev. B 49, 2148 (1994).

${ }^{5}$ C. L. Kane, K. A. Matveev, and L. I. Glazman, Phys. Rev. B 49, 2253 (1994).

${ }^{6}$ I. Affleck and A. W. W. Ludwig, J. Phys. A 27, 5375 (1994).

${ }^{7}$ Y. Oreg and A. M. Finkel'stein, Phys. Rev. B 53, 10928 (1996); Phys. Rev. Lett. 76, 4230 (1996).

${ }^{8}$ D. H. Lee and J. Toner, Phys. Rev. Lett. 69, 3378 (1992).

${ }^{9}$ A. Furusaki and N. Nagaosa, Phys. Rev. Lett. 72, 892 (1994).

${ }^{10}$ A. Schiller and K. Ingersent, Phys. Rev. B 51, 4676 (1995).

${ }^{11}$ P. Fröjdh and H. Johannesson, Phys. Rev. Lett. 75, 300 (1995); Phys. Rev. B 53, 3211 (1996).

${ }^{12}$ C. L. Kane and M. P. A. Fisher, Phys. Rev. Lett. 68, 1220 (1992); Phys. Rev. B 46, 15233 (1992).

${ }^{13}$ K. A. Matveev, D. Yue, and L. I. Glazman, Phys. Rev. Lett. 71, 3351 (1993).

${ }^{14}$ M. Ogata and H. Fukuyama, Phys. Rev. Lett. 73, 468 (1994).

${ }^{15}$ P. Fendley, A. W. W. Ludwig, and H. Saleur, Phys. Rev. Lett. 74, 3005 (1995).

${ }^{16}$ S. Tarucha, T. Honda, and T. Saku, Solid State Commun. 94, 413 (1995).

${ }^{17}$ F. P. Milliken, C. P. Umbach, and R. A. Webb, Solid State Commun. 97, 309 (1996).
${ }^{18}$ A. M. Chang, L. N. Pfeiffer, and K. W. West, Phys. Rev. Lett. 77, 2538 (1996).

${ }^{19}$ F. Lesage, H. Saleur, and S. Skorik, Phys. Rev. Lett. 76, 3388 (1996); G. Gómez-Santos, ibid. 76, 4223 (1996).

${ }^{20}$ Y. L. Liu, Phys. Lett. A 233, 143 (1997).

${ }^{21}$ M. Fabrizio and A. O. Gogolin, Phys. Rev. B 51, 17827 (1995).

${ }^{22}$ S. Eggert, H. Johannesson, and A. Mattsson, Phys. Rev. Lett. 76, 1505 (1996).

${ }^{23}$ M. Fabrizio and A. O. Gogolin, Phys. Rev. Lett. 78, 4527 (1997); Y. Oreg and A. M. Finkel'stein, ibid. 78, 4528 (1997).

${ }^{24}$ A. Luther and I. Peschel, Phys. Rev. B 9, 2911 (1974).

${ }^{25}$ V. J. Emery, in Highly Conducting One-Dimensional Solids, edited by J. T. Devreese et al. (Plenum Press, New York, 1979); J. Sólyom, Adv. Phys. 28, 201 (1979).

${ }^{26}$ F. D. M. Haldane, J. Phys. C 14, 2585 (1981).

${ }^{27}$ J. Schwinger, Phys. Rev. 128, 2425 (1962).

${ }^{28}$ D. K. K. Lee and Y. Chen, J. Phys. A 21, 4155 (1988).

${ }^{29}$ A. Schmit, Phys. Rev. Lett. 51, 1506 (1983); M. P. A. Fisher and W. Zwerger, Phys. Rev. B 32, 6190 (1985).

${ }^{30}$ R. Egger and H. Grabert, Phys. Rev. Lett. 75, 3505 (1995); 79, 3463 (1997).

${ }^{31}$ V. J. Emery and S. Kivelson, Phys. Rev. B 46, 10812 (1992).

${ }^{32}$ M. Fabrizio, A. O. Gogolin, and P. Nozières, Phys. Rev. B 51, 16 088 (1995).

${ }^{33}$ Y. L. Liu, Phys. Rev. Lett. 79, 293 (1997). 